\title{
Training eldercare workers in mental healthcare
}

\author{
Gwen Li $\underline{\operatorname{Sin}}^{1}$, MBBS, MMed, Donald $\underline{Y e o}^{2}$, PsyD, Hwan Jing $\underline{K o h}^{3}$, MSc, Jimmy $\underline{L e}^{4}$, MBBS, MMed, Li Ling $\underline{\operatorname{Ng}}{ }^{5}$, MBBS, MMed
}

INTRODUCTION A significant proportion of older persons who require support or care services have mental health concerns. There is a need to equip frontline eldercare workers with the necessary skills in caring for older persons with mental illnesses. The Community Psychogeriatric Programme aims to support community eldercare providers with training and consultation. We sought to measure eldercare workers' perceived levels of stress and knowledge about caring for older persons with mental illness before (pre-workshop), immediately after (post-workshop) and three months after (three-month follow-up) they underwent standardised training workshops on dementia and depression.

METHODS Participants who attended two four-hour workshops on dementia and depression were recruited for the study. Their knowledge of topics was evaluated pre-workshop, post-workshop and at three-month follow-up. Perceptions of working with older persons with mental health problems were rated pre-workshop and at three-month follow-up.

RESULTS A total of 71 staff members from various eldercare centres participated in the study, of which 51 ( $71.8 \%$ ) were women. At three-month follow-up, there was a significant change in most measures on Ryden's Perception Scale $(p<0.05)$. There were significant improvements in knowledge scores for dementia and depression $(p<0.001)$. Knowledge gains after the workshop were maintained at three-month follow-up.

CONCLUSION Training of eldercare workers in mental healthcare is helpful for knowledge improvement and altering perceptions of caring for older persons. With continued support from mental health professionals, such training could contribute to better care for this vulnerable population.

Keywords: dementia, depression, mental health, training eldercare workers

\section{INTRODUCTION}

The population of older persons (aged 60 years and above) is expected to reach a proportion of $21.1 \%$ of the world's population by 2050, or more than double from 841 million people in 2013 to more than two billion. ${ }^{(1)}$ The population of adults aged over 65 years in Singapore increased to $13.7 \%$ in 2016. ${ }^{(2)}$ A recent Singapore-based study by Subramaniam et al found that the prevalence of 10/66 dementia was $10 \%$ in the older adult population, while the prevalence of dementia according to Diagnostic and Statistical Manual of Mental Disorders (4th edition) criteria was $4.6 \%{ }^{(3)}$ There are major social and economic consequences to these trends, as old-age support ratios continue to fall. In Singapore, home- and communitybased care, as well as intermediate and long-term care services, have mushroomed over the recent years following efforts by governmental agencies to cater to the rising need for such services. As a result, a significant number of eldercare workers are required. Most eldercare workers are trained to care for the physical and health-related needs of elderly people, including the provision of physical rehabilitation and maintenance exercises, and social and recreational activities. However, healthcare workers tend to be less confident when handling elderly people with mental illnesses. Given recent trends, it is necessary to equip our eldercare workers with lasting skills and confidence when it comes to caring for elderly people with mental illnesses.

In a review of the effectiveness of staff training programmes, McCabe et al suggested that skills training for nursing staff increases their knowledge and skill base and reduces the number of behaviour problems among residents. ${ }^{(4)}$ There is also an indication that training may lead to greater staff satisfaction and have an impact on turnover rates. Refresher training sessions are then needed to sustain changes in staff knowledge and skills. Feldt and Ryden measured the effects of a training programme on nursing assistants' perceptions of behaviour and their chosen management techniques when dealing with aggressive behaviours. They found that training enabled nursing assistants to choose more effective interventions for managing aggressive behaviours. ${ }^{(5)}$ Palmer and Withee looked at the perceptions of nursing home staff after a training session on the nature of work with assaultive residents, the frequency and severity of problem behaviours, and communication patterns around the issue of behaviour management. ${ }^{(6)}$ On Ryden's Perception Scale (RPS), they found that all parameters shifted to positive ends of the scale, with the exception of the rating of 'very time-consuming'. Proctor et al found that levels of reported psychological distress were shown to increase significantly among the control group, who received no training and support, when compared to the intervention group, who received a six-month intervention programme devised for developing skills in individual care planning. In their study, $57 \%$ of control patients reached 'caseness' threshold on the General Health Questionnaire. ${ }^{(7)}$ Moniz-Cook et al argued that staff development programmes reduce burnout and improve 'therapeutic reciprocity' in residential care staff. ${ }^{(8)}$

The Community Psychogeriatric Programme (CPGP) in Singapore was started in 2007 under the Ministry of Health,

\footnotetext{
${ }^{1}$ Department of Psychiatry, Singapore General Hospital, ${ }^{2}$ KALL Psychological and Counselling Services Pte Ltd, Novena Medical Centre, ${ }^{3}$ Alzheimer's Disease Association (Singapore), ${ }^{4}$ Department of General Psychiatry 1, Institute of Mental Health, ${ }^{5}$ Department of Psychological Medicine, Changi General Hospital, Singapore

Correspondence: Dr Gwen Li Sin, Consultant, Department of Psychiatry, Singapore General Hospital, Academia, Level 3, 20 College Road, Singapore 169856. sin.gwen.li@singhealth.com.sg
} 
Table I. Outline and learning objectives of the workshops.

\begin{tabular}{ll}
\hline Workshop on dementia (4 hr) & Workshop on depression (4 hr) \\
\hline $\begin{array}{l}\text { Overview } \\
\text { - An overview of the diagnosis, assessment and management of dementia }\end{array}$ & $\begin{array}{l}\text { Overview } \\
\text { - An overview of the diagnosis, assessment and } \\
\text { management of depression in older persons }\end{array}$ \\
$\begin{array}{l}\text { Recognising dementia } \\
\text { - Identify the signs and symptoms of dementia } \\
\text { - Understand the clinical presentation of dementia through case examples } \\
\text { - Learn to use rating instruments to assess cognitive and memory } \\
\text { functions }\end{array}$ & $\begin{array}{l}\text { - Identify the signs and symptoms of depression } \\
\text { - Understand the clinical presentation of depression } \\
\text { through case examples }\end{array}$ \\
$\begin{array}{l}\text { Helping and supporting older persons with dementia } \\
\text { - Learn to manage older persons with challenging behaviours, } \\
\text { such as wandering, agitation and aggression }\end{array}$ & $\begin{array}{l}\text { Helping and supporting older persons with depression } \\
\text { - Learn how to recognise and assist an older person who } \\
\text { is suicidal }\end{array}$ \\
\hline
\end{tabular}

Singapore, to fund and provide community mental health services for early detection and treatment of psychogeriatric disorders. ${ }^{(9)}$ One of the CPGP's key objectives is engagement of community eldercare services through regular consultation, training and continued support of community eldercare providers.

In this present study, we set out to measure community eldercare workers' perceived levels of stress and confidence when caring for elderly people with mental illness before and three months after undergoing standardised training workshops on dementia and depression. We hypothesised that after attending the two workshops on dementia and depression in the elderly, participants would have reduced levels of stress and increased knowledge of caring for elderly people with mental illness.

\section{METHODS}

Training material for the workshops was developed jointly by the multidisciplinary CPGP team. Team members were certified in classroom-based facilitated training, courseware and standardised training sessions. Table I describes the outline and objectives of each workshop.

Training workshops began with a survey of the perceptions of eldercare workers regarding looking after elderly people with mental illness (using RPS), and a pre-workshop assessment of participants' knowledge of the topic. This was followed by didactic teaching, case discussions and group discussions. Trainers were psychiatrists, mental health nurses, psychologists and other allied mental healthcare professionals from the CPGP. Each workshop lasted four hours and took place either on the same day or within a space of two weeks. The session ended with a summary of key points and a reassessment of participants' knowledge of the topic.

Eldercare agencies in partnership with the CPGP were invited to send their staff for these training workshops on mental healthcare for elderly people between 2009 and 2011. The agencies consisted of daycare providers for elderly people or nursing homes. English-literate participants who attended the training workshops were invited to participate in the study. Written informed consent was obtained from all participants. The study received ethical approval from the Centralised Institutional Review Board, Singapore Health Services, Singapore. Participants completed a data form for basic demographic information. This included information on vocation, years of experience in eldercare, age, ethnicity and nationality.

The Mental Health Literacy Survey (MHLS) is a ten-item truefalse questionnaire on knowledge-based statements pertaining to the workshop topics. It was administered thrice - before the workshop (pre-workshop), immediately after the workshop (post-workshop) and three months after the workshop (threemonth follow-up). The MHLS was jointly designed by the team to highlight the main teaching points of each course.

$\mathrm{RPS}^{(5)}$ is a six-item semantic differential (Likert) scale to measure participants' perception of their experience of caring for elderly people with mental illness. Participants were asked to choose a point on the scale between each set of words that best described their experience of caring for elderly people with mental illness. This was administered pre-workshop and at three-month follow-up. A post-training survey, administered at three-month follow-up, involved anecdotal responses and was an open-ended questionnaire that gathered participants' views of how they had benefitted from the workshop.

Descriptive statistics were used to report characteristics of the study sample. Repeated measures were examined using nonparametric tests, such as Wilcoxon signed-rank test and Friedman test for two- and three-time point measurements, respectively. Statistical significance was set at two-tailed $p<0.05$. All statistical analyses were conducted using IBM SPSS Statistics version 20.0 (IBM Corp, Armonk, NY, USA).

\section{RESULTS}

Participants had a multiethnic distribution and women (71.8\%) were overrepresented (Table II). A majority of participants were care staff $(60.6 \%)$ and had daily contact $(56.3 \%)$ with mentally ill persons. All 71 participants who attended the workshop consented to participate in the study. Data was missing for one participant. At the three-month time point, 5 (7.1\%) of the 70 participants were lost to follow-up.

At three-month follow-up, there was a significant change in most measures on RPS (Table III). Participants reported that caring 
for elderly people with mental illness was easier $(p<0.001)$, less time-consuming $(p=0.002)$, more satisfying $(p=0.023)$ and safer $(p<0.001)$. A summary of the knowledge scores of participants pre- and post-workshop and at three-month follow-up is shown in Table IV. There was a significant improvement in knowledge scores for dementia and depression across the three time points ( $p<0.001$ for all time points). In the post-hoc analysis, gains in knowledge scores for dementia were evident between preworkshop and post-workshop ( $p<0.001)$, and between postworkshop and three-month follow-up ( $p=0.014)$. However, while significant knowledge gains were noted between pre- and post-workshop scores $(p<0.001)$ for depression, no such gain was evident between post-workshop and at three-month followup $(\mathrm{p}=0.589)$.

In the post-training survey, participants indicated that they were generally more confident in managing elderly people with mental illness after the workshops. Many participants reported being able to understand patients better, indicating that they were 'always questioning and probing why they behave in a certain way'. Another frequent response was that they were 'more confident in dealing with elderly with mental illness'. Some participants mentioned that they were 'more patient and able to give the elderly more time to respond'. Participants felt that they had 'improved communication with the elderly with greater understanding of their emotional needs'. They also cited having better knowledge of how to detect dementia and depression, and interact with elderly people with dementia or depression. However, one participant indicated that it was difficult to apply the new knowledge due to lack of manpower and constraints of time and space.

\section{DISCUSSION}

Our findings suggest that training workshops help to improve and sustain knowledge for at least three months after they are conducted. Eldercare workers' perceptions of caring for elderly people with mental illness shifted significantly to the positive end of the spectrum for four out of six RPS subscales. Of note, our study participants found it less time-consuming to care for elderly people with mental illness after the workshops than before. This is in contrast with Palmer and Withee's finding that nursing home staff who underwent training did not find it less time-consuming to care for the elderly after the training session(s) than before. ${ }^{(6)}$ This disparity in findings could be partly due to differences in the population of elderly people being managed by the staff in the two studies. Eldercare workers in our sample were from diverse eldercare settings and worked with elderly people who were less medically ill compared to participants from Palmer and Withee's study. ${ }^{(6)}$

After the workshops, no improvements were noted among our participants in scores on the rewarding-thankless and challengingboring subscales on the RPS. It is possible that participants may have misunderstood the challenging-boring subscale, as the term 'challenging' may have a negative connotation, especially given that 'challenging behaviours in dementia' was used during the workshop to describe problematic behaviour. Similarly, for
Table II. Characteristics of participants $(n=71)$.

\begin{tabular}{|c|c|}
\hline Variable & No. (\%) \\
\hline Age $(y r)^{*}$ & $46.3 \pm 12.8$ \\
\hline \multicolumn{2}{|l|}{ Gender } \\
\hline Men & $20(28.2)$ \\
\hline Women & $51(71.8)$ \\
\hline \multicolumn{2}{|l|}{ Ethnicity } \\
\hline Chinese & $42(59.2)$ \\
\hline Malay & $8(11.3)$ \\
\hline Indian & $4(5.6)$ \\
\hline Filipino & $9(12.7)$ \\
\hline Burmese & $7(9.9)$ \\
\hline Other & $1(1.4)$ \\
\hline \multicolumn{2}{|l|}{ Vocation } \\
\hline Nursing staff & $5(7.0)$ \\
\hline Allied health staff & $5(7.0)$ \\
\hline Care staff & $43(60.6)$ \\
\hline Administrative staff & $10(14.1)$ \\
\hline Volunteer & $8(11.3)$ \\
\hline \multicolumn{2}{|l|}{ Education ${ }^{+}$} \\
\hline Primary & $1(1.4)$ \\
\hline Secondary & $31(43.7)$ \\
\hline Pre-university & $13(18.3)$ \\
\hline Tertiary & $24(33.8)$ \\
\hline \multicolumn{2}{|c|}{ Contact with mentally ill persons ${ }^{*}$} \\
\hline Daily & $40(56.3)$ \\
\hline Weekly or monthly & $9(12.7)$ \\
\hline Several times a year & $8(11.3)$ \\
\hline Never & $13(18.3)$ \\
\hline
\end{tabular}

*Data presented as mean \pm standard deviation. †Missing data for two participants. ¥Missing data for one participant.

the rewarding-thankless subscale, the measure was unlikely to have changed following training if participants considered returns for providing care in terms of external rewards. Indeed, perceptions and stress levels of eldercare staff are important but understudied components that contribute to staff burnout and increased staff turnover, ${ }^{(10)}$ and they may affect the quality of care provided to eldercare patients. In view of the above, it is important that teaching staff recognise mental health issues commonly seen among elderly people and impart skills for responding to symptoms of depression and dementia that would be beneficial to both the carer and patient in the long run.

The strengths of our study included its high response and low dropout rates. The inclusion of community eldercare agencies in our study was another strength, as these care providers are an important cluster engaged in the overall efforts to detect and manage mental illness among elderly people in the community, but are rarely studied.

There were some limitations to our study. First, participants were not randomly selected and there was no control group, as this was a naturalistic study. Second, the study sample may not be entirely representative of other professional care staff working with elderly people having mental illness. Third, some groups received varying degrees of follow-up support from the CPGP 
Table III. Comparison of pre- and post-workshop scores of participants using Ryden's Perception Scale.

\begin{tabular}{lccc}
\hline Subscale & \multicolumn{2}{c}{ Mean \pm standard deviation score } & p-value \\
\cline { 2 - 3 } & Pre-workshop $(\mathbf{n}=\mathbf{7 0})^{*}$ & 3-month follow-up $(\mathbf{n}=\mathbf{6 5})+$ & $<0.001^{\S}$ \\
\hline Very difficult-very easy & $3.86 \pm 1.16$ & $4.68 \pm 0.97$ & 0.932 \\
Rewarding-thankless & $3.43 \pm 1.48$ & $3.46 \pm 1.31$ & $0.002^{\S}$ \\
Time-consuming-quickly done & $3.59 \pm 1.23$ & $4.40 \pm 1.10$ & $0.023^{\S}$ \\
Frustrating-satisfying & $4.36 \pm 1.37$ & $4.92 \pm 0.96$ & 0.170 \\
Challenging-boring & $3.19 \pm 1.27$ & $3.57 \pm 1.27$ & $<0.001^{\S}$ \\
Frightening-safe & $4.13 \pm 1.34$ & $5.09 \pm 0.95$ & \\
\hline
\end{tabular}

*Data was missing for one participant. +Five participants were lost to follow-up. $\neq$ p-value was calculated using Wilcoxon signed-rank test. §p < 0.05 was considered statistically significant.

Table IV. Comparison of pre-workshop, post-workshop and three-month follow-up knowledge scores of participants.

\begin{tabular}{lccc}
\hline Variable & \multicolumn{3}{c}{ Mean \pm standard deviation score } \\
\cline { 2 - 3 } & Pre-workshop & Post-workshop & 3-month follow-up \\
\hline Dementia & $6.57 \pm 1.92$ & $7.55 \pm 1.42$ & $8.33 \pm 1.62$ \\
& $(n=68)$ & $(n=69)$ & $(n=60)$ \\
Depression & $6.86 \pm 1.78$ & $8.14 \pm 1.47$ & $8.28 \pm 1.34$ \\
& $(n=59)$ & $(n=58)$ & $(n=53)$ \\
\hline
\end{tabular}

Data was missing and the corresponding no. of participants is provided. ${ }^{*} \mathrm{p}$-value was calculated using Friedman test. $\dagger p<0.05$ was considered statistically significant.

team within the study duration as part of ongoing community support work, which could have confounded the study findings. This support could have been in the form of consultations for management of challenging clients, case discussions, additional training workshops and other informal contact. It is possible that participants who received additional support could have gained knowledge that altered their perceptions as a result of the extra interaction with mental healthcare professionals. This might also have accounted for some of the significant gains noted in knowledge scores for dementia vis-à-vis the post-workshop and three-month follow-up time points.

A fourth limitation was that there were different trainers for some workshops. Although the workshop materials and processes were identical, we could not account for differences in trainers' styles and group dynamics that might have affected learning among different participant groups. Fifth, we also do not discount the possibility of response bias. Participants may have completed the questionnaires with only favourable responses, as they did not want to be perceived as not having benefitted from the workshop or create a negative perception of their work. We attempted to reduce this bias by de-identifying the survey forms. In addition, participants were reassured that their responses were confidential and would not be provided to anyone outside the training team. While the improvement in knowledge subsequent to training was statistically significant, it was challenging to measure if this represented a clinically meaningful change. Nevertheless, we found that these gains in knowledge were sustained for up to three months after the workshops.

Future studies in dementia care could include the Approaches to Dementia Questionnaire ${ }^{(11)}$ to specifically look at attitudes of eldercare workers toward persons with dementia. As Well Being Profiling ${ }^{(12)}$ and Dementia Care Mapping ${ }^{(13)}$ are becoming increasingly popular and powerful approaches for measuring specific aspects of dementia care outcomes in daycare facilities, these could be useful tools in determining whether such training workshops translate to improving the quality of care in similar settings.

In conclusion, the training of eldercare workers managing older persons with mental illness plays an important role in altering their perceptions of caring for such patients. Knowledge gained can be sustained after one session, and with continued support from mental health professionals, this could contribute to improved care of older persons with mental illness in the long term.

\section{ACKNOWLEDGEMENTS}

The authors would like to acknowledge all members of the Community Psychogeriatric Programme team who helped with training and data collection, as well as Mary Chan and Ling Mingquan for their invaluable administrative support. The study was financially supported by grants received from the Clinical Trials and Research Unit at Changi General Hospital, Singapore.

\section{REFERENCES}

1. United Nations Department of Economics and Social Affairs Population Division. World Population Ageing 2013. Available at: http://www.un.org/en/development/ desa/population/publications/pdf/ageing/WorldPopulationAgeing2013.pdf. Accessed June 8, 2017.

2. The National Population and Talent Division, Singapore. Population in Brief 2016. Available at: https://www.nptd.gov.sg/PORTALS/0/HOMEPAGE/ HIGHLIGHTS/population-in-brief-2016.pdf. Accessed June 8, 2017

3. Subramaniam M, Chong SA, Vaingankar JA, et al. Prevalence of dementia in people aged 60 years and above: results from the WiSE Study. J Alzheimers Dis 2015; 45:1127-38.

4. McCabe MP, Davison TE, George K. Effectiveness of staff training programs for behavioral problems among older people with dementia. Aging Ment Health 2007; 11:505-19.

5. Feldt KS, Ryden MB. Aggressive behavior. Educating nursing assistants. J Gerontol Nurs 1992; 18:3-12.

6. Palmer AC, Withee BM. Dementia care: effects of behavioral intervention training on staff perceptions of their work in veterans' nursing home. Geriatr Nurs 1996; 17:137-40.

7. Proctor R, Stratton-Powell H, Tarrier N, Burns A. The impact of training and support on stress among care staff in nursing and residential homes for the elderly. J Ment Health 1998; 7:59-70. 
8. Moniz-Cook E, Millington D, Silver M. Residential care for older people: job satisfaction and psychological health in care staff. Health Soc Care Community 1997; 5:124-33

9. Sin GL, Ng LL. The Community Psychogeriatric Programme: a pilot project in the eastern sector of Singapore. Singapore Med J 2011; 52:463-5.

10. Cohen-Mansfield J. Stress in nursing home staff: a review and a theoretical model. J Appl Gerontol 1995; 14:444-66.
11. Lintern T, Woods B, Phair L. Before and after training: a case study of intervention. J Dementia Care 2000; 8:15-7.

12. Bradford Dementia Group. The Bradford well-being profile. Bradford: University of Bradford, 2008.

13. Fossey J, Lee L, Ballard C. Dementia Care Mapping as a research tool for measuring quality of life in care settings: psychometric properties. Int J Geriatr Psychiatry 2002; 17:1064-70. 